\title{
Acronyms and Initialisms
}

\section{Costa Rica}

AI Autonomous institution

AMCHAM-Costa Rica American Chamber of Commerce of Costa Rica ANDE Asociación Nacional de Educadores; National Association of Educators

ANEIT Asociación Nacional de Exportadores de la Industria Textil; National Association of Textile Industry Exporters

ANEP Asociación Nacional de Empleados Públicos y Privados; National Association of Public and Private Employees

APSE Asociación de Profesores de Segunda Enseñanza; Association of Secondary Education Teachers

CADEXCO Cámara de Exportadores de Costa Rica; Costa Rican Chamber of Exporters

CDI Consejo de Defensa de la Institucionalidad; Council for the Defense of Institutionality

CEFSA Consultores Económicos y Financieros, SA; Economic and Financial Consultants, SA

CICR Cámara de Industrias de Costa Rica; Chamber of Industry of Costa Rica

CINDE Coalición Costarricense de Iniciativas en Desarrollo; Costa Rican Investment Promotion Agency

CINPE Centro Internacional de Política Económica para el Desarrollo Sostenible; International Center for the Political Economy of Sustainable Development

CODESA Corporación Costarricense de Desarrollo, SA; Costa Rican Development Corporation, SA 
COMEX Ministerio de Comercio Exterior, Foreign Trade Ministry

CONCAUSA Consejo Empresarial para las Negociaciones Comerciales Centroamérica-Estados Unidos; Business Council for the Central America-U.S. Trade Negotiations

CTRN Confederación de Trabajadores Rerum Novarum; Rerum Novarum Confederation of Workers

FECON Federación Conservacionista; Conservationist Federation

ICE Instituto Costarricense de Electricidad; Costa Rican Electrical Institute

MIDEPLAN Ministerio de Planificación Nacional y Política Económica; Ministry of National Planning and Political Economy

ML Movimiento Libertario; Libertarian Movement Party

PAC Partido de Acción Ciudadana; Citizen Action Party

PAE Programa de ajuste estructural; structural adjustment program

PEN Programa Estado de la Nación; State of the Nation Program

PLN Partido de Liberación Nacional; National Liberation Party

PND Plan Nacional de Desarrollo; National Development Plan

PROCOMER Promotora de Comercio Exterior; Trade Promotion Agency PUSC Partido de Unidad Social Cristiana; Social Christian Unity Party

TSE Tribunal Supremo Electoral; Supreme Electoral Tribunal

UCCAEP Unión Costarricense de Cámaras y Asociaciones de la Empresa Privada; Costa Rican Union of Private Sector Chambers and Associations

UCR Universidad de Costa Rica; University of Costa Rica

UNA Universidad Nacional; National University

UPANACIONAL Unión de Productores Agropecuarios-Nacional; National Agricultural Producers Union

El Salvador

ADES Asociación de Desarrollo Económico y Social; Economic and Social Development Association

AMCHAM-El Salvador American Chamber of Commerce of El Salvador AMPES Asociación de Medianos y Pequeños Empresarios Salvadoreños; Salvadoran Association of Small and Medium-Sized Business Owners

ANEP Asociación Nacional de la Empresa Privada; National Private Enterprise Association 
ARENA Alianza Republicana Nacionalista; Nationalist Republican Alliance

ASI Asociación Salvadoreña Industria; Association of Salvadoran Industry

ASIC Asociación Salvadoreña de la Industria de la Confección; Salvadoran Apparel Industry Association

BCR Banco Central de Reserva; Central Reserve Bank

CAMAGRO Cámara Agropecuaria y Agroindustrial de El Salvador; Agricultural and Agroindustrial Chamber of El Salvador

CCIES Cámara de Comercio e Industria de El Salvador; Chamber of Commerce and Industry of El Salvador

CD Cambio Democrático; Democratic Change Party

CDU Centro Democrático Unido; United Democratic Center

CEDES Conferencia Episcopal de El Salvador; Episcopal Conference of El Salvador

CEICOM Centro de Investigación sobre Inversión y Comercio; Investment and Trade Research Center

CEMUJER Instituto de Estudios de la Mujer "Norma Virginia Guirola de Herrera"; Norma Virginia Guirola de Herrera Institute of Women's Studies

CES Consejo Económico y Social; Social and Economic Council

CISPES Committee in Solidarity with the People of El Salvador

COEXPORT Corporación de Exportadores de El Salvador; Corporation of Salvadoran Exporters

CONAES Consejo Nacional de Empresarios Salvadoreños; National Council of Salvadoran Business Owners

CONAMUS Coordinadora Nacional de Mujeres Salvadoreñas; National Coordinating Committee of Salvadoran Women

CONFRAS Confederación de Federaciones de la Reforma Agraria Salvadoreña; Confederation of Salvadoran Agrarian Reform Cooperatives

CORDES Fundación para la Cooperación y el Desarrollo Comunal de El Salvador; Foundation for Cooperation and Community Development of El Salvador

CRIPDES Asociación de Comunidades Rurales para el Desarrollo de El Salvador; Rural Communities Association for Salvadoran Development

CSTS Coordinadora Sindical de Trabajadoras(es) de El Salvador; Trade Union Coordinating Committee of Salvadoran Workers 
ENEPASA Asociación Intermunicipal de Energía para El Salvador; Intermunicipal Energy Association of El Salvador

FENAPES Federación Nacional de la Pequeña Empresa de El Salvador; National Federation of Small Enterprises of El Salvador

FESPAD Fundación de Estudios para la Aplicación del Derecho; Foundation for the Study of the Application of Law

FIMES Federación Independiente de Microempresarios Salvadoreños; Independent Federation of Salvadoran Microenterprise Owners

FISDL Fondo de Inversión Social para el Desarrollo Local; Fund for Social Investment for Local Development

FMLN Frente Farabundo Martí para la Liberación Nacional; Farabundo Martí National Liberation Front

FSC Foro de la Sociedad Civil; Civil Society Forum

FUNDE Fundación Nacional para el Desarrollo; National Development Foundation

FUSADES Fundación Salvadoreña para el Desarrollo Económico y Social; Salvadoran Social and Economic Development Foundation

FUSADES-DEES FUSADES-Departamento de Estudios Económicos y Sociales; Economic and Social Studies Department

GANA Gran Alianza por la Unidad Nacional; Grand Alliance for $\mathrm{Na}-$ tional Unity

IUDOP Instituto Universitario de Opinión Pública; University Public Opinion Institute

MARN Ministerio del Medio Ambiente y Recursos Naturales; Ministry of the Environment and Natural Resources

MPR12 Movimiento Popular de Resistencia-12 de Octubre; October 12 Popular Resistance Movement

ODASP Oficina de Apoyo al Sector Productivo para las Negociaciones Comerciales; Office of Assistance for the Business Sector in Trade Negotiations

PCN Partido de Conciliación Nacional; National Conciliation Party

PDC Partido Demócrata Cristiano; Christian Democrat Party

PDDH Procuraduría para la Defensa de los Derechos Humanos; Office of the Human Rights Defense Attorney

RST Red Sinti Techán; Sinti Techán Network

SHARE Salvadoran Humanitarian Aid, Research and Education Foundation

TSE Tribunal Supremo Electoral; Supreme Electoral Tribunal

UCA Universidad Centroamericana José Simeón Cañas; Central American University 
UES Universidad de El Salvador; University of El Salvador UNES Unidad Ecológica Salvadoreña; Salvadoran Ecological Unity

\section{Nicaragua}

ALBANISA ALBA de Nicaragua, SA; ALBA of Nicaragua, SA

ALN Alianza Liberal Nicaragüense; Nicaraguan Liberal Alliance AMCHAM-Nicaragua American Chamber of Commerce of Nicaragua APEN Asociación Nicaragüense de Productores y Exportadores; Nicaraguan Producer and Exporter Association

APRE Alianza para la República; Alliance for the Republic

BANADES Banco Nacional de Desarrollo; National Development Bank

BCN Banco Central de Nicaragua; Central Bank of Nicaragua

CA Complementary Agenda

CACONIC Cámara de Comercio de Nicaragua; Nicaraguan Chamber of Commerce

CARUNA Caja Rural Nacional, RL; National Rural Fund

CEI Centro de Estudios Internacionales; International Studies Center

CINASE Centro de Investigación y Asesoría Socioeconómica; Center for Socioeconomic Research and Consulting

CIPRES Centro para la Promoción, la Investigación y el Desarrollo Rural y Social; Center for Rural and Social Promotion, Research, and Development

CNZF Corporación Nicaragüense de Zonas Francas; Nicaraguan Free Trade Zones Corporation

CONPES Consejo Nacional de Planificación Económica Social; National Social and Economic Planning Council

COSEP Consejo Superior de la Empresa Privada; Superior Council of Private Enterprise

CPC Consejo del Poder Ciudadano; Citizen Power Council

CST-JBE Confederación Sindical de Trabajadores-"José Benito Escobar" (formerly Central Sandinista de Trabajadores); José Benito Escobar Union Federation of Workers

FENACOOP Federación Nacional de Cooperativas Agropecuarias y Agroindustriales; National Federation of Agricultural and Agroindustrial Cooperatives

FIDEG Fundación Internacional para el Desafío Económico Global; International Fund for Global Economic Challenge 
FSLN Frente Sandinista de Liberación Nacional; Sandinista National Liberation Front

FUNIDES Fundación Nicaragüense para el Desarrollo Económico y Social; Nicaraguan Foundation for Social and Economic Development

GRUN Gobierno de Reconciliación y Unidad Nacional; Government of Reconciliation and National Unity

INIDE Instituto Nacional de Información de Desarrollo de Nicaragua; Nicaraguan National Development Information Institute

MAGFOR Ministerio Agropecuario y Forestal; Ministry of Agriculture and Forestry

MIFIC Ministerio de Fomento de Industria y Comercio; Ministry of Industry and Trade Promotion

MRS Movimiento Renovador Sandinista; Sandinista Renovation Movement

PLC Partido Liberal Constitucionalista; Liberal Constitutionalist Party

PNDH Plan Nacional para el Desarrollo Humano; National Human Development Plan

UNAG Unión Nacional de Agricultores y Ganaderos; National Union of Farmers and Ranchers

UNAPA Unión Nacional Agropecuaria de Productores Asociados; National Agricultural Union of Associated Producers

UNO Unión Nacional Opositora; National Opposition Union

\section{General}

AFL-CIO American Federation of Labor-Congress of Industrial Organizations

AFT Aid for Trade

ALBA Alianza Bolivariana para los Pueblos de Nuestra América; Alliance for the Peoples of Our America

AMCHAM American Chamber of Commerce

ASC

Alianza Social Continental (also HSA)

BCIE Banco Centroamericano de Integración Económica; Central American Bank of Economic Integration

BIT Bilateral investment treaty

CACM Central America Common Market

CAFTA Central America Free Trade Agreement (also CAFTA-DR) 
CAFTA-DR Dominican Republic-Central America Free Trade Agreement

CBI Caribbean Basin Initiative

C-CAA Caribbean-Central American Action

CCT Conditional cash transfer

CECA Consejo Empresarial Centroamericano; Central American Business Council

CELAM Consejo Episcopal Latinoamericano; Latin American Episcopal Council

CIEL Center for International Environmental Law

CSOP Civil Society Outreach Program

ECLAC Economic Commission on Latin America and the Caribbean

EIA Environmental impact assessment

EPZ Export processing zone

EZLN Ejército Zapatista de Liberación Nacional; Zapatista Army of National Liberation

FDI Foreign direct investment

FECAGRO Federación Centroamericana de Cámaras Agropecuarias y Agroindustriales; Central American Federation of Agriculture and Agroindustry Chambers

FLACSO Facultad Latinoamericana de Ciencias Sociales; Latin American School of Social Sciences

FTA Free trade agreement

FTAA Free Trade Area of the Americas

GAO Government Accountability Office (U.S.)

GATT General Agreement on Tariffs and Trade

GDP Gross domestic product

GSP Generalized System of Preferences

HIPC Heavily indebted poor country

HSA Hemispheric Social Alliance (also ASC)

IAHRC Inter-American Human Rights Commission

ICSID International Centre for Settlement of Investment Disputes

IDB Inter-American Development Bank (also IADB)

IFI International financial institution

ILO International Labour Organization

IMF International Monetary Fund

IMF-WEO IMF-World Economic Outlook

INCAE Instituto Centroamericano de Administración de Empresas;

Central American Business Administration Institute 
INGO International nongovernmental organization

Iniciativa CID Iniciativa Mesoamericana Comercio, Integración y Desarrollo; Mesoamerican Trade, Integration, and Development Initiative (also CID)

INTAL Instituto para la Integración de América Latina y el Caribe; Institute for the Integration of Latin America and the Caribbean

IO International organization

IPR Intellectual property rights

IPS Institute for Policy Studies

ISI Import-substitution industrialization

LAPOP Latin American Public Opinion Project

MCC Millennium Challenge Corporation

MIPYMES Micro, pequeñas y medianas empresas; micro, small, and medium-sized businesses

MSME Micro, small, and medium-sized enterprise

NAFTA North American Free Trade Agreement

NGO Nongovernmental organization

OAS Organization of American States

OCMAL Observatorio de Conflictos Mineros de América Latina; Latin American Mining Conflicts Observer

PDVSA Petróleos de Venezuela, SA

PELA Proyecto Élites Parlamentarias Latinoamericanas; Parliamentary Elites in Latin America

PETROCARIBE Acuerdo de Cooperación Energética entre Venezuela y Países del Caribe y Centroamérica; Energy Cooperation Agreement between Venezuela and Caribbean and Central American Countries

PNUD Programa de las Naciones Unidas para el Desarrollo; United Nations Development Programme (also UNDP)

PPP Plan Puebla-Panamá

RMALC Red Mexicana de Acción Frente al Libre Comercio; Mexican Action Network on Free Trade

SAPRIN Structural Adjustment Participatory Review International Network

SICA Sistema de Integración Centroamericana; Central American Integration System

SIECA Secretaría de Integración Económica Centroamericana; Central American Economic Integration Secretariat

TCB Trade capacity building

TINA There is no alternative 
TLC Tratado de Libre Comercio

TNC Transnational corporation

TPA Trade promotion authority

TRIM Trade related investment measure

TRIP Trade related intellectual property

UNCTAD United Nations Conference on Trade and Development

UNDP United Nations Development Programme (also PNUD)

USAID U.S. Agency for International Development

USTR U.S. Trade Representative

WB/WDI World Bank, World Development Indicators

WOLA Washington Office on Latin America

WTO World Trade Organization 
THIS PAGE INTENTIONALLY LEFT BLANK 